\title{
Can utility optimization explain the demand for structured investment products?
}

\author{
Hens, Thorsten ; Rieger, Marc Oliver
}

\begin{abstract}
In this paper, we first show that for classical rational investors with correct beliefs and constant absolute or constant relative risk aversion, the utility gains from structured products over and above a portfolio consisting of the risk-free asset and the market portfolio are typically much smaller than their fees. This result holds irrespectively of whether the investors can continuously trade the risk-free asset and the market portfolio at no costs or whether they can just buy the assets and hold them to maturity of the structured product. However, when considering behavioural utility functions, such as prospect theory, or investors with incorrect beliefs (arising from probability weighting or probability misestimation), the utility gain can be sizable.
\end{abstract}

DOI: https://doi.org/10.1080/14697688.2013.823512

Posted at the Zurich Open Repository and Archive, University of Zurich

ZORA URL: https://doi.org/10.5167/uzh-95114

Journal Article

Accepted Version

Originally published at:

Hens, Thorsten; Rieger, Marc Oliver (2014). Can utility optimization explain the demand for structured investment products? Quantitative Finance, 14(4):673-681.

DOI: https://doi.org/10.1080/14697688.2013.823512 


\title{
Can utility optimization explain the demand for structured investment products?
}

\author{
Thorsten Hens* \\ Marc Oliver Rieger ${ }^{\dagger}$
}

July 2, 2013

\begin{abstract}
In this paper, we first show that for classical rational investors with correct beliefs and constant absolute or constant relative risk aversion, the utility gains from structured products over and above a portfolio consisting of the risk-free asset and the market portfolio are typically much smaller than their fees. This results holds irrespectively of whether the investors can continuously trade the risk-free asset and the market portfolio at no costs or whether they can just buy the assets and hold them to maturity of the structured product. However, when considering behavioral utility functions, such as prospect theory, or investors with incorrect beliefs (arising from probability weighting or probability misestimation), the utility gain can be sizable.
\end{abstract}

JEL classification: G11,D14,C61,D03,D18.

Keywords: structured products, utility maximization, behavioral finance, prospect theory

${ }^{*}$ Swiss Finance Institute Professor at the University of Zurich, Plattenstrasse 32,CH8032 Zurich, Switzerland; and Norwegian School of Economics, Helleveien 30, NO-5045, Bergen, Norway; thorsten.hens@bf.uzh.ch

${ }^{\dagger}$ Professor of Banking and Finance, University of Trier, Germany; mrieger@uni-trier.de 


\section{Introduction}

Structured investment products, also known as equity- or index-linked notes, are financial derivatives, the payoff of which at maturity depends on one or more standard assets (mostly stocks or stock indices). Most of these products are issued by banks and are targeted to retail investors, which can be observed from the way they are marketed. Structured products have gained substantial popularity in recent years with retail investors in the US, but even more so in Europe and East Asia. The market capitalization of the Swiss market alone is more than 200 billion Euros. In some countries (such as Germany and Switzerland), approximately $6 \%$ of all financial assets are held in structured products (Rieger 2009). Considering the huge variety of structured products ( see e.g. the Swiss Derivatives Map), nearly every possible payoff diagram exists in the market.

For example, barrier reverse convertibles are very popular products ${ }^{1}$ that offer a coupon much higher than the risk-free rate, and they pay back the initial investment, unless at some point before maturity the underlying hits a barrier, i.e., depreciates more than the pre-specified value. If a barrier is hit, the holder of the product obtains a share of the underlying at maturity, instead of his or her originally invested capital. The payoff of a barrier reverse convertible is path dependent. Hence, for the same value of the underlying at maturity, the structured product delivers payoffs that depend on the path that the underlying took to that value.

The general motivation of our study was to determine why the demand for various types of structured products is so high. To answer to this question, we first investigate whether, from a rational perspective, structured products make sense ${ }^{2}$. Instead of evaluating specific structured products from the

\footnotetext{
${ }^{1}$ Their market share in the US was $18.37 \%$ (in 2006), and it was as high as $30.5 \%$ in Switzerland (in 2007).

${ }^{2}$ As is well know from (Merton 1971) in an ideal world with normally distributed returns
} 
long list of existing structured products, we take an indirect approach. We compute the optimal structured product for a class of utility functions and then assess whether, in this best possible case, there is a significant utility increase. If this is not the case, we can conclude that none of the existing structured products benefits the customers. More precisely, we calculate the utility gain (in terms of certainty equivalents) of structured products for expected utility maximizers with correct beliefs. We call beliefs "correct" if they coincide with the market returns.

Our main finding is that the utility increase of the best possible structured product, over and above a simple portfolio consisting of risk-free assets and the market portfolio, is only 5 to 10 basis points, bp, and thus rather small - too little compared with the usual margins (100 - 200 bp) paid on structured products - not to mention a compensation for the counterparty risk that the buyer of a structured product takes. It thus seems like one needs to look beyond standard finance assumptions to explain the popularity of structure products. To shed light on this issue, we model investors as maximizing prospect theory type utility functions and we also analyze the effect of incorrect beliefs on the demand for structured products. We show that for behavioral investors maximizing prospect theory utility, the gains from structured products can be sizable because prospect theory investors are loss averse and prefer to take risks rather than being certain to lose. Additionally, structured products might be the optimal choice for investors with incorrect beliefs, e.g., when they are overconfident.

Previous academic research on structured products has nearly exclusively focused on the issuer's perspective, mostly within the context of option

and no transaction cost structured products are not needed since continuously trading the market portfolio and the risk-free asset would be sufficient to achieve the highest utility of an investor with constant relative risk aversion and correct beliefs. We generalize these findings to any return distribution and to less frequent trading. The latter allows the application to retail investors that cannot trade continously 
pricing and hedging. Elaborate pricing techniques have been developed for increasingly complicated structured products. In addition to this enormous technical literature, several interesting empirical studies have recently been published on the actual market prices of structured products in the US (Benet, et al. 2006) and in Europe (Wilkens, et al. 2003, Grünbichler \& Wohlwend 2005, Stoimenov \& Wilkens 2005, Szymanowska, et al. 2007, Wilkens \& Stoimenov 2007, Wallmeier \& Diethelm 2008) . However, no comprehensive studies have yet attempted to understand which types of structured products are attractive for private investors and for what motives, although this topic has recently drawn much attention from a number of papers addressing specific puzzles in this field: Henderson \& Pearson (2007) studied empirical differences in popular payoff diagrams between structured products with single stocks and stock market indices as underlying assets. Branger \& Breuer (2007) and Breuer \& Perst (2007) computed the potential utility gain for CRRA investors for a number of structured products on the German market. Bernard, et al. (2007) studied the characteristics of optimal capital-protected products for various investor preferences. In a recent study, Henderson \& Pearson (2011) analyzed a specific class of products (so-called SPARQs) offered on the US market ${ }^{3}$. They concluded that "issuing firms ... introduce complexity to exploit uninformed investors" a conclusion that fits very well into our broader picture. The mispricing of SPARQs (the difference between the issuing price and the theoretical market value) was so large that their expected return was lower than that of a

\footnotetext{
${ }^{3}$ SPARQ is the acronym for Stock Participation Accreting Redemption Quarterly-pay Securities. These are securities issued that mimic the payoff of a stock but carry the counterparty risk of the investment bank issuing the SPARQ.
} 
fixed-interest investment ${ }^{4}$ ! Shefrin \& Statman (1993) and Shefrin \& Statman (2000) analyzed some specific structured products from a behavioral perspective: covered calls, premium bonds, lottery bonds and LYONS (a combination of bonds and calls). Moreover, Shefrin (2008) provides good intuition, based on attractive examples, on which structured products are optimal for SP/A decision makers ${ }^{5}$. Thus, the literature contains many interesting results on structured products, but no systematic comparisons of the usefulness of structured products for utility maximizers can be found. We hope that our systematic optimization approach will introduce more structure into the debate. Our paper is organized as follows. In the next section, we summarize the canonical two-period model for structured products that we will use throughout this paper. In Section 3, we analyze structured products from a rational investor's perspective (using von NeumannMorgenstern utilities with correct beliefs), while in Section 4, we extend our analysis to behavioral utility maximization in the sense of prospect theory and to incorrect beliefs. Section 5 concludes the paper.

\section{A utility maximization model for structured products}

In this section, we introduce a canonical two-period utility maximization model for structured products. The choice of a two-period model means that investors buy structured products with the intention to hold them until maturity. This is indeed typically the case for structured investment

\footnotetext{
${ }^{4}$ Note, however, that simple structured products in Germany or Switzerland are mispriced by less than $1 \%$, and thus their expected return is still greater than the fixed interest rate (compare the above-mentioned studies on mispricing, e.g., Wilkens \& Stoimenov (2007).)

${ }^{5} \mathrm{SP} /$ A theory goes back to Lopes (1987) and Lopes \& Oden (1999). It can be considered a generalization of the safety first principle of Roy (1952), allowing for probability weights as, e.g. prospect theory does.
} 
products. Our model follows closely the standard framework that can be found, e.g., in studies by Duffie (2001) or Leland (1980). We assume that the market is complete for product designers, i.e., the bank ${ }^{6}$, and that investors are "price-takers", i.e., they do not influence prices by themselves (or as groups of identical investors). We also assume that the investor does not hold assets other than the structured product. Of course, if the structured product were considered to be an addition on top of a portfolio of other assets, then its use would be derived in connection with the other assets returns, in the same way that a put option on a stock market index is useful to protect a portfolio of individual stocks temporarily. However, none of the advertisements of structured products indicates this use. They are really considered standalone investments that are separate from other assets.

The investor's preferences are described by a utility function $u: \mathbb{R} \rightarrow \mathbb{R}$. Given our application, we can assume that the payoff of the products is bounded below by zero, i.e., we cannot lose more money than we have invested. This limitation will ensure that the set on which we maximize is compact. Moreover, we assume standard technical conditions on $u$, e.g., that $u$ is continuous. The market return is described by a probability measurement, $p$. The case in which the investor's beliefs differ from the market belief or the true probabilities is discussed in Section 4.2. The payoff of the structured product can be modeled as a function $y: \mathbb{R} \rightarrow \mathbb{R}^{7}$ We assume here for simplicity that the product's return depends solely on the market

\footnotetext{
${ }^{6}$ Note that the investors are not sufficiently knowledgeable to compose structured products by themselves, nor would it be feasible at all for them to do so, because dynamic hedging is often required for their construction. This is why the bank can earn a margin by selling the structured product and hedging it on the market.

${ }^{7}$ Given the complexity of many structured investment products on the market, one might wonder why we can assume that the structured product in the above model is a function of the market return at maturity; barriers are, e.g., not included into this category. In the two-period model this is, however, not a problem because, as Rieger (2011) has shown, optimal products must be functions of the underlying.
} 
index. There are, in fact, many structured products on single stocks or baskets of stocks, but the theoretical model needed to study them would be rather similar. Thus, an investor wants to maximize

$$
U(y):=\int_{\mathbb{R}} u(y(x)) d p(x)
$$

over all $y \in L_{\ell p}^{1}(\mathbb{R})$, i.e. over all functions measurable with respect to $\ell p$, where $\ell$ is the likelihood ratio and $x$ is the state space variable. A standard budget constraint leads to the side constraint

$$
\int_{\mathbb{R}} y(x) \ell(x) d p(x)=R
$$

We assume for the moment that $\ell$ is given by the capital asset pricing model, CAPM, $\ell(x)=a-b x$, with $a=1+b \mu$ and $b=(\mu-R) / \sigma^{2}$, where $\mu$ and $\sigma^{2}$ are the mean and variance of the market portfolio, respectively, $x$ is its payoff and $R$ is the payoff of the risk-free asset. We assume, if not otherwise specified, that $\mu=1.09$ (i.e., an average stock market return of $9 \%$ ), $\sigma=0.20$, and $R=1.04$ (i.e., a risk-free rate of $4 \%$ ). In this case, state prices in the CAPM are positive up to a return of approximately $+100 \%{ }^{8}$ For our considerations, however, it is usually sufficient to assume $\ell$ to be continuous and decreasing (e.g., in the case of Black-Scholes) ${ }^{9}$. Choosing the CAPM as the pricing model for an investor with preferences other than mean-variance is, of course, not at odds with the CAPM market pricing assumptions because we study an individual decision problem and not market equilibrium. We mention that even if we were to close the model and needed to derive asset prices as well, the validity of the CAPM would not be in contradiction to violations of the two-fund separation property. For example, heterogeneous beliefs or uncorrelated optimization mistakes give

\footnotetext{
${ }^{8}$ Negative state prices might arise in the CAPM because of the famous mean-variance paradox; see Jarrow \& Madan (1997) for details.

${ }^{9}$ In consumption-based models, $\ell$ is a decreasing function of the market return as a direct consequence of decreasing marginal utility of wealth.
} 
rise to heterogeneous portfolios, while at the market level, the security market line property of the CAPM can still hold (Bossaert 2002, Chiarella, et al. 2007, Gerber \& Hens 2006).

\section{Rational reasons for structured products}

Let us assume that the investor has rational preferences and correct beliefs, i.e., he or she follows the expected utility approach by von Neumann \& Morgenstern (1944). Let us also assume for the moment that its utility function, $u$, is continuously differentiable, strictly increasing and strictly concave. This case has been studied previously (Leland 1980, Pliska 1984, Kramkov \& Schachermayer 1999, Carr \& Madan 2001). A straightforward variational approach leads to the optimality condition

$$
y(x)=v^{-1}(\lambda \ell(x))
$$

where $v:=u^{\prime}$ and $\lambda$ is a Lagrange parameter that must be chosen such that (2) holds.

We study two prominent examples of specific utility functions, in which the payoff function of the optimal structured product can be given explicitly. In both examples, we first derive the shape of the optimal structured product. We see that optimal structured products deliver a payoff structure that is different from that of classical portfolios (including only the market portfolio and a risk-free asset). The effort of computing such an optimally structured product and hedging it can, in practice, not be neglected; therefore, the natural question arises of whether it is worth the effort. How large is the potential utility improvement? ${ }^{10}$

To answer this question, we then compute the expected utility of an optimally structured product and the expected utility of the optimal mix be-

\footnotetext{
${ }^{10}$ Branger \& Breuer (2007) considered a similar question for a selection of typically structured products.
} 
tween the market portfolio and the risk-free asset. We translate both values into certainty equivalent interest rates, i.e., the (hypothetical) risk-free asset that would have the same expected utility as the product. We then consider the difference between both certainty rates and compare it with the gain in certainty rates that can be achieved by a classical portfolio over a risk-free investment.

This analysis shows how good the "second-order approximation" (the classical two-fund portfolio) is, compared to the "higher-order approximation" (the optimally structured product). In this way, we want to answer a couple of questions of practical relevance: is the first (classical) step of improvement significant and the second step (caused by the structured product) negligible? Or are they equally important? Under what circumstances is the additional potential improvement of structured products particularly large?

\section{Power utility}

Let us first consider the power utility function $u(x)=x^{\alpha} / \alpha$. The power utility function is one of the standard workhorse models in finance. It has many attractive properties, such as a constant relative risk aversion so that as, for example, the percentage allocation of wealth among risky assets keeps constant when wealth changes (Gollier 2004), e.g., due to fluctuations of the returns. Additionally, strong aggregation results can be derived if the agents share the same degree of risk aversion (Rubinstein 1974). We first derive the shape of the payoff function of optimal structured products in the case of power utility. Let $u(x):=\frac{1}{\alpha} x^{\alpha}$ with $\alpha<1, \alpha \neq 0$. Then the optimal structured product is given by

$$
y(x)=\frac{C}{\ell(x)^{\frac{1}{1-\alpha}}},
$$

where $\ell$ is the likelihood ratio, $x$ is the state space variable and $C$ is a positive constant, as shown by the computation in the appendix. Obtaining 
precise intuition for this formula is more difficult. It is, however, plausible that an increase in the likelihood ratio of a state leads to a decrease of the payoff $y$, as would be expected: an optimal product yields lower payoffs in "expensive" states than in "cheap" states. The precise relation is influenced by the degree of risk aversion. If the risk aversion is high (i.e., $\alpha \rightarrow-\infty$ ), $y$ becomes nearly constant (risk-free investment). In the risk-neutral limit (i.e., $\alpha \rightarrow 1$ ), $y$ becomes very steep. We can also see from this formula that if the likelihood ratio $\ell(x)$ is affine in $x$ (which is the case in CAPM), the structured product is an increasing and convex function of the underlying.

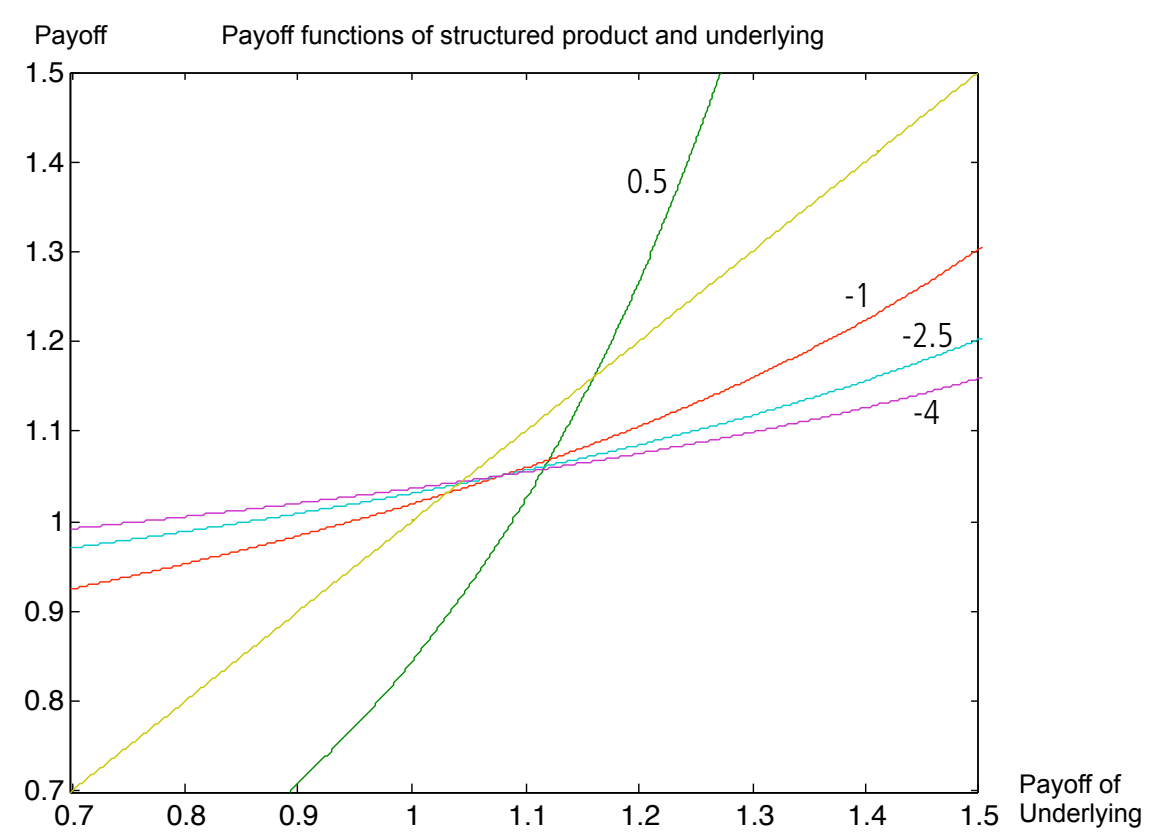

Figure 1: Optimally structured product for CRRA-investors with $\alpha=$ $-4,-2.5,-1,0.5$.

The optimally structured products for expected utility investors with this utility function on a CAPM market ${ }^{11}$ are shown in Fig. 1, for some values

\footnotetext{
${ }^{11}$ Here and in the following examples, we suppose, if not stated differently, $R=1.04$, $\sigma=0.2$ and $\mu=1.09$. These values are typical values for non-crisis times. Our following observations are quite robust to changes in these parameters, as we will see below.
} 
of $\alpha$. In the past, values of $\alpha$ of approximately -2 or -3 have been proposed based on empirical evidence. $\alpha=1$ would correspond to risk-neutral behavior.

We see that the optimal payoff function of the product is strictly convex. It features "smooth" capital protection and an increasing participation in gains. For low risk aversion, the slope of the optimal payoff profile becomes (partially) larger than one (its Delta at the money is larger than one), which can be understood as short-selling in the risk-free asset. As long as the bankruptcy constraint $(y \geq 0)$ is satisfied, there is nevertheless no theoretical reason that an investor could not buy such a product. Combining the riskfree asset with the market portfolio in a buy and hold way would generate a linear payoff. However, as can be seen from Figure 1 the deviation from linearity is not large. So looking at the payoff diagram one might already conjecture that the utility gain is not high.

Next, we compute the utility gain relative to a classical portfolio composed of the risk-free asset and the market portfolio. To compute the optimal classical portfolio, we compute the utility of all portfolios with a proportion of $\theta$ invested in the market portfolio and $(1-\theta)$ invested into risk-free assets, where $\theta \in\{0,0.01, \ldots, 0.99,1\} .{ }^{12}$ Then, we choose the $\theta$ yielding the largest expected utility.

The results of this computation are summarized in Table 1.

We see that the optimally structured product gives an improvement, but it is not as large as the "first step", i.e., the improvement induced by the

\footnotetext{
${ }^{12}$ Negative values of $\theta$ would correspond to a short-selling of the market portfolio, while $\theta>1$ would correspond to taking a loan to have leverage on the stock market investment. Both cases would obviously violate the bankruptcy constraint; thus, we do not consider them here. For nearly risk-neutral investors, the optimally structured product would include states with negative return, thus essentially enabling the investor to short-sell. This is the only reason why the optimally structured product seems to be beneficial to investors with very low risk aversion.
} 
Table 1: Utility comparison for an investor with a classical CRRA utility with $\alpha=-2$.

\begin{tabular}{lc} 
Investment & Certainty equivalent \\
\hline Fixed interest (4\%) & $4.00 \%$ \\
Market portfolio (optimal classical) & $5.09 \%$ \\
Optimal structured product & $5.16 \%$
\end{tabular}

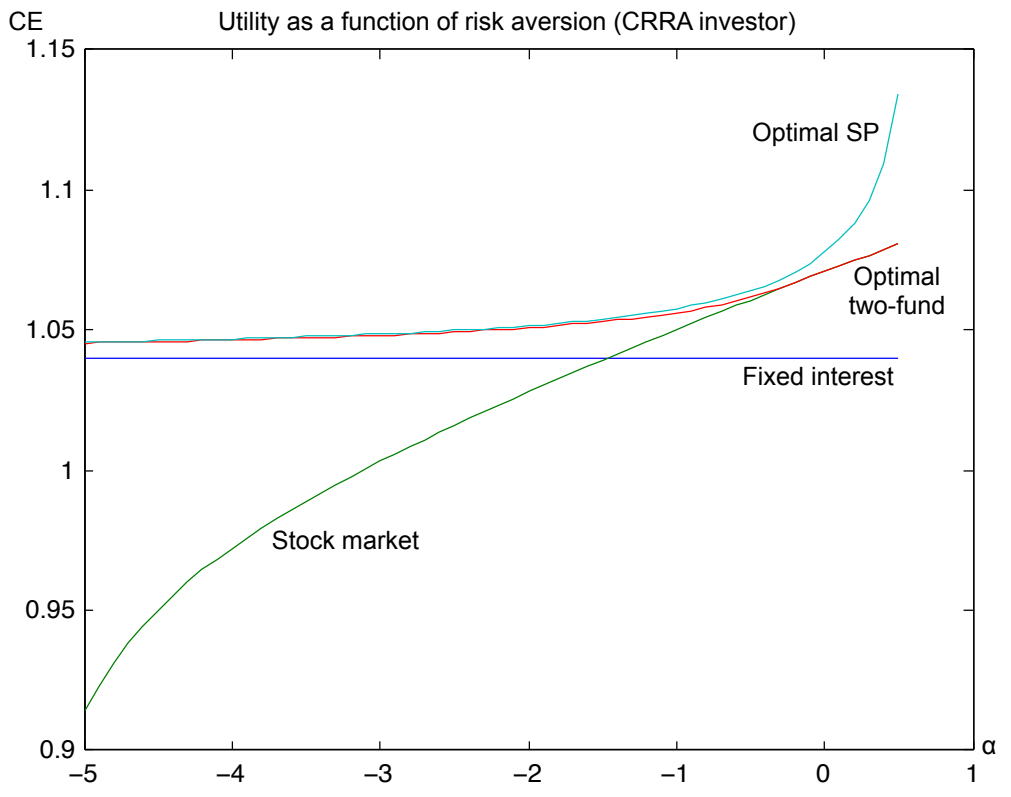

Figure 2: Utility for CRRA varying between $\alpha=-5$ and $\alpha=1$ (as measured by the certainty equivalent interest rate) of stock, risk-free asset (4\% return), optimal two-fund portfolio and optimal structured product. The utility gain by the structured product is small. 
classical mean-variance portfolio theory. The improvement over the classical portfolio is only seven basis points. The smallness of this improvement is not due to a specific choice of $\alpha$. Very much to the contrary, Fig. 2, Fig. 3 and Fig. 4 show that the improvement is in fact small for a wide range of choices for risk aversion $\alpha$, volatility $\sigma$ and interest $r$.

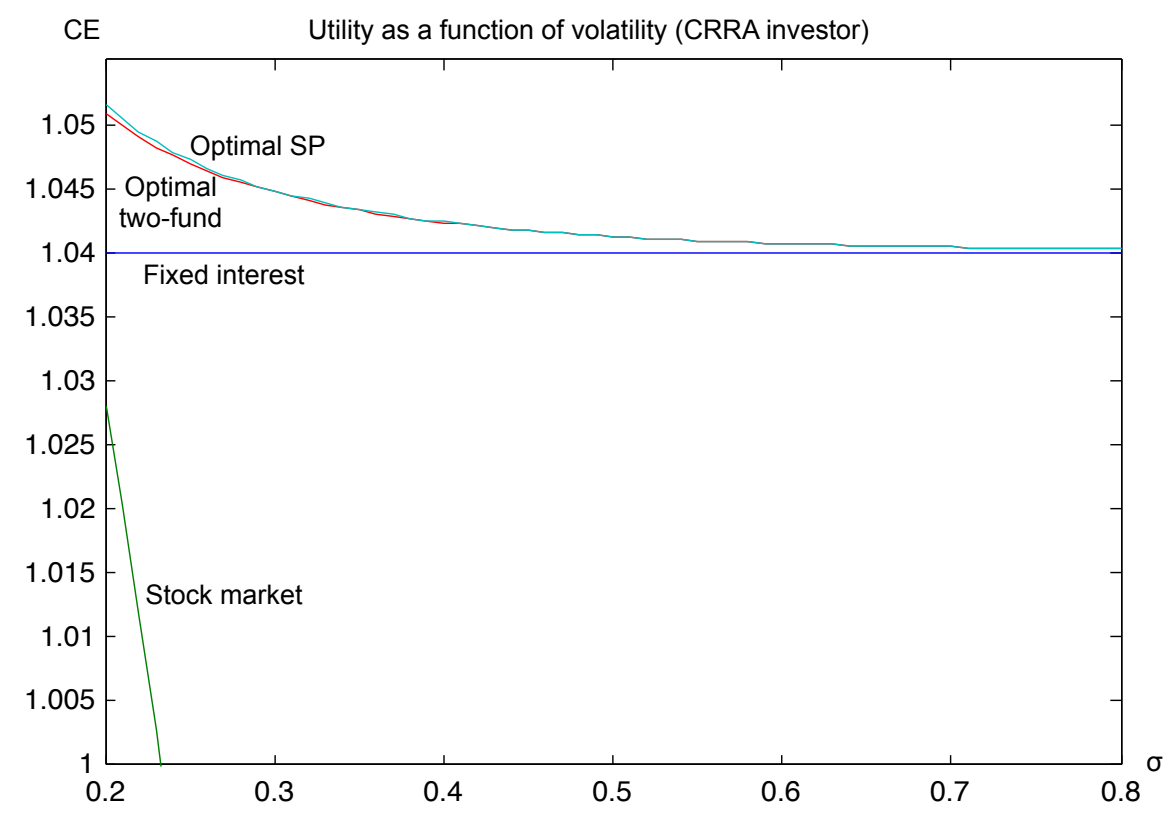

Figure 3: Utility for CRRA of $\alpha=-2$ (as measured by the certainty equivalent interest rate) of stock, risk-free asset (4\% return), optimal two-fund portfolio and optimal structured product when the volatility of the risky asset varies. The utility gain by the structured product is small.

\section{Exponential utility}

Exponential utility is another workhorse in finance because it shares properties similar to those of the power utility. In this case, however, the $a b$ solute risk aversion is constant, and the number of risky assets in the asset allocation does not fluctuate with wealth (Gollier 2004). Additionally, it 


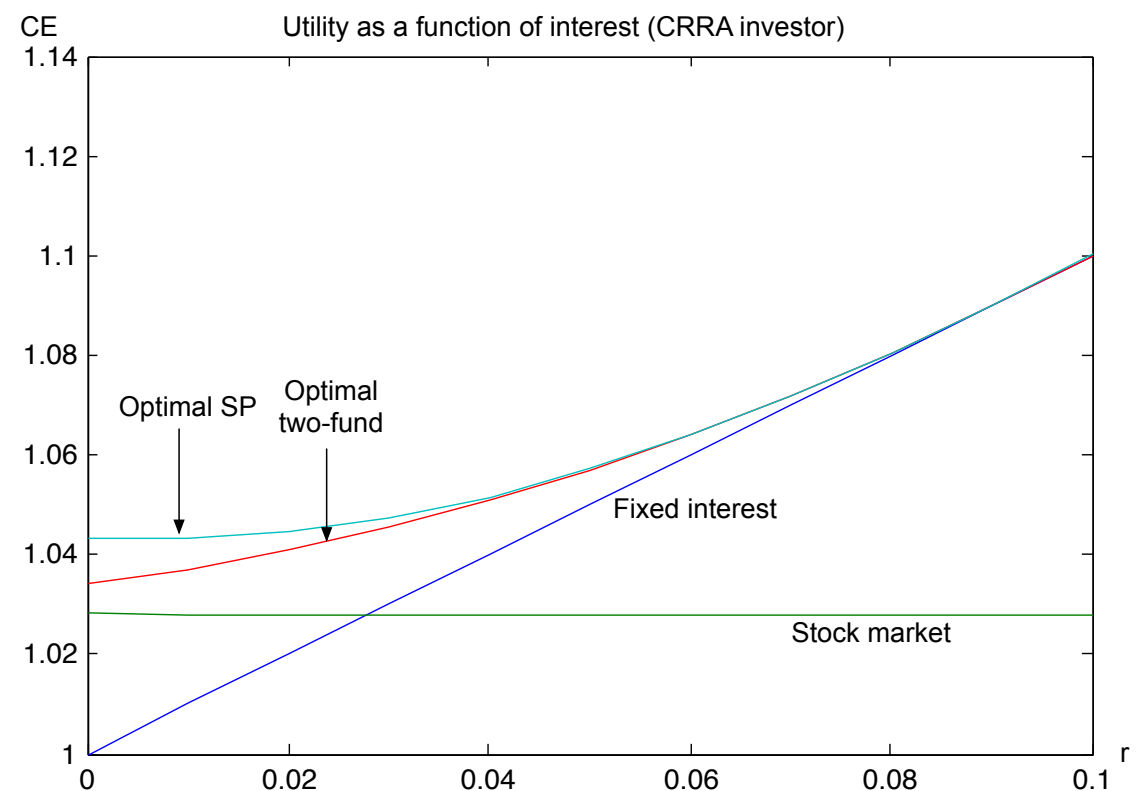

Figure 4: Utility for CRRA of $\alpha=-2$ (as measured by the certainty equivalent interest rate) of stock, risk-free asset (4\% return), optimal two-fund portfolio and optimal structured product when the risk-free rate varies. The utility gain by the structured product is small. 
allows for good aggregation results (Rubinstein 1974). In the case of exponential utility, the shape of the payoff diagram is derived as follows. Let $u(x):=-\frac{1}{\alpha} e^{-\alpha x}$. Then, the optimally structured product in the CAPM case is

$$
y(x)=R-\frac{1}{\alpha}(\ln (a-b x)-C),
$$

where $C$ is a positive constant (see appendix). Again, $y$ is an increasing and strictly convex function of $x$ (in the CAPM case). As in the case of CRRA, we now compute the utility gain relative to a classical portfolio composed of the risk-free-asset and the market portfolio.

Table 2: Utility comparison for an investor with a classical CARA utility with $\alpha=10$ (a typical choice for a CARA utility).

\begin{tabular}{lc} 
Investment & Certainty equivalent \\
\hline Fixed interest (4\%) & $4.00 \%$ \\
Optimal classical portfolio & $4.31 \%$ \\
Optimal structured product & $4.32 \%$
\end{tabular}

Here the improvement is again very small (only $0.012 \%$ ). This does not come as a big surprise, as the optimal structured product is close to linear, as our numerical computations show. The result is very similar if we vary the absolute risk aversion.

We have seen that for CRRA and CARA investors with correct beliefs on a CAPM market, the utility improvement that structured products can maximally provide is far too small to make them attractive. Replacing CAPM with Black-Scholes as the pricing model in the numerical computation does not change the picture either, which is not very surprising because this replacement would not change that the optimal payoff function does not deviate much from a straight line. 
From (Merton 1971) it is well known that our result needs to hold if the rational investor could continuously trade the risk-free asset and the market portfolio in a market with normally distributed returns and no transaction costs. However, our two period model may also describe a situation in which investors buy assets in the first period and hold them to the second period (maturity of the structured product). In contrast to the continuous time model, in this case introducing small transaction costs will not destroy the result. Moreover, the result we obtain does not require normally distributed returns.

Summing up, we conclude that the improvements of structured products for classical rational investors with correct beliefs are somewhere between tiny and small and are definitely less than the typical costs (which can be in the order of $1 \%$ or more even in competitive markets, such as Germany or Switzerland $)^{13}$.

This finding might be the reason why the optimally structured product for expected utility maximizers is not offered on the market. The utility gain over a simple portfolio, consisting of a risk-free asset and a market index (which leads to a linear payoff as a function of the underlying), is tiny, while the replication costs might be high for the optimal product, which is strictly convex. Structured products - much ado about nothing? We need to look for alternative models that can explain the demand for structured investment products. In the following section, we therefore discuss potential behavioral reasons that can explain investors' decisions to invest into structured products.

\footnotetext{
${ }^{13}$ One might wonder whether rational investors buy structured products to improve an existing portfolio of assets. This is however not logical since - as we have shown, rational investors do not need to hold portfolios other than the risk-free asset and the market portfolio to obtain the highest possible utility. That is to say holding a portfolio (different to the risk-free asset and the market portfolio) so that a structured product makes sense is an unecessarily complicated construction for a rational investor.
} 


\section{Behavioral reasons for structured products}

In many areas of finance, it has been very fruitful to abandon the assumption of complete rationality and to model decision problems based on psychological findings. Indeed, this type of research has become its own sub-discipline in finance, known as behavioral finance. We show that behavioral finance is also a fruitful route for understanding structured products. While behavioral finance highlights many departures from the rational paradigm, for the understanding of most structured products, we evoke only a few of those biases: loss aversion, gambling to avoid sure losses, weighting and misestimation of probabilities and over- and underconfidence. For a recent survey on behavioral finance reviewing the evidence for and the consequences of these and other biases see (Barberis \& Thaler 2003).

\subsection{Loss aversion and risk taking to avoid sure losses}

We now study whether behavioral preferences, as in the prospect theory of Kahneman \& Tversky (1979), can better explain the demand for structured products. In prospect theory, the utility $u$ depends on a reference point (e.g., the current wealth level): it is steeper for losses than for gains (loss aversion), and it is concave for gains and strictly convex for small losses (gambling to avoid sure losses). Tversky \& Kahneman (1992) have suggested to model these features by the followuing piecewise power value function:

$$
u(x):= \begin{cases}-\lambda(-x)^{\beta}, & \text { for } x<0 \\ x^{\alpha}, & \text { for } x \geq 0 .\end{cases}
$$

If not stated otherwise, we assume the parameter values that Kahneman \& Tversky (1979) found in their emprical study: $\alpha=\beta=0.88$ and $\lambda=2.25$. What optimally structured product could such a model describe for an investor? Because $u$ is, in this case, not strictly concave, we cannot apply 
the aforementioned results (Pliska 1984, Kramkov \& Schachermayer 1999). However, using the results in Rieger (2012), we can replace $u$ with its concavification, i.e., the smallest concave function larger or equal to $u$, and then follow the same computations as before. The only difficulty is that the inverse of the derivative is not everywhere defined because the derivative of the concavification can be constant. This, however, simply corresponds to a jump in the inverse $y$, as observed using an approximation argument. With this concavification, we can compute optimally structured products also for non-concave utility functions, e.g., in the case of prospect theory.

We can think here of an investor who is (like most investors) averse to losses but who does not distinguish much between small and large losses. This lack of distinction leads to risk-seeking behavior in losses. Depending on the amount of loss-aversion, $\lambda$ as a smaller or larger amount of capital protection becomes optimal.

Figure 5 shows the optimal solution in this case for various values of lossaversion. We see from this figure that the optimally structured product for a prospect theory utility function offers capital protection up to a certain size of losses, and it delivers a more than proportional participation in high gains.

Similar payoff structures indeed exist in the market for structured products (compare, e.g., outperformance bonus certificates).

When we consider a prospect theory investor (without probability weighting) with a utility function, as in Tversky \& Kahneman (1992) with $\alpha=\beta=$ 0.88 , his or her utility improvement for various levels of loss aversion $\lambda$ is considerable; see Fig. 6. Keeping loss aversion constant as 2.25, but varying the level of risk taking in losses, denoted by $\beta$, does not lead to substantial utility gains (see Fig. 7).

A closer looks shows that the utility gain becomes, in particular, substantial for low loss aversion. This finding is, at first glance, surprising, but it can 


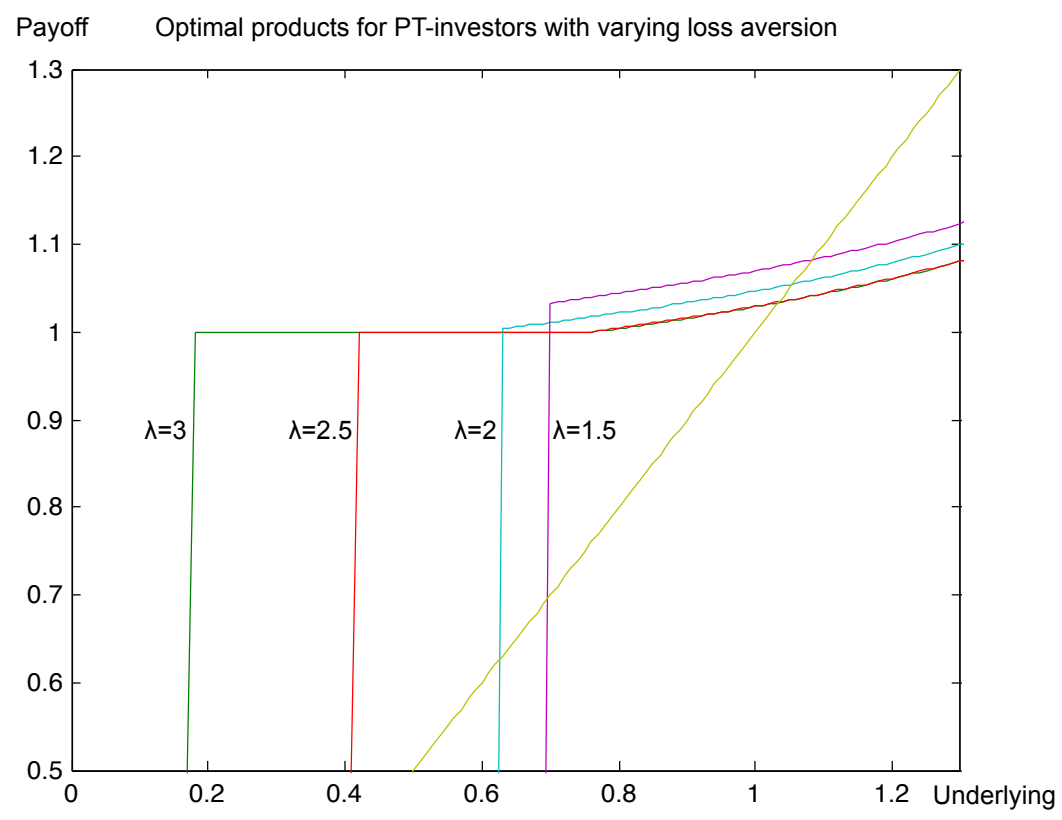

Figure 5: Optimal structured products for investors with a piecewise power prospect theory utility with $\alpha=\beta=0.88$ and $\lambda=1.5,2,2.5,3$. 


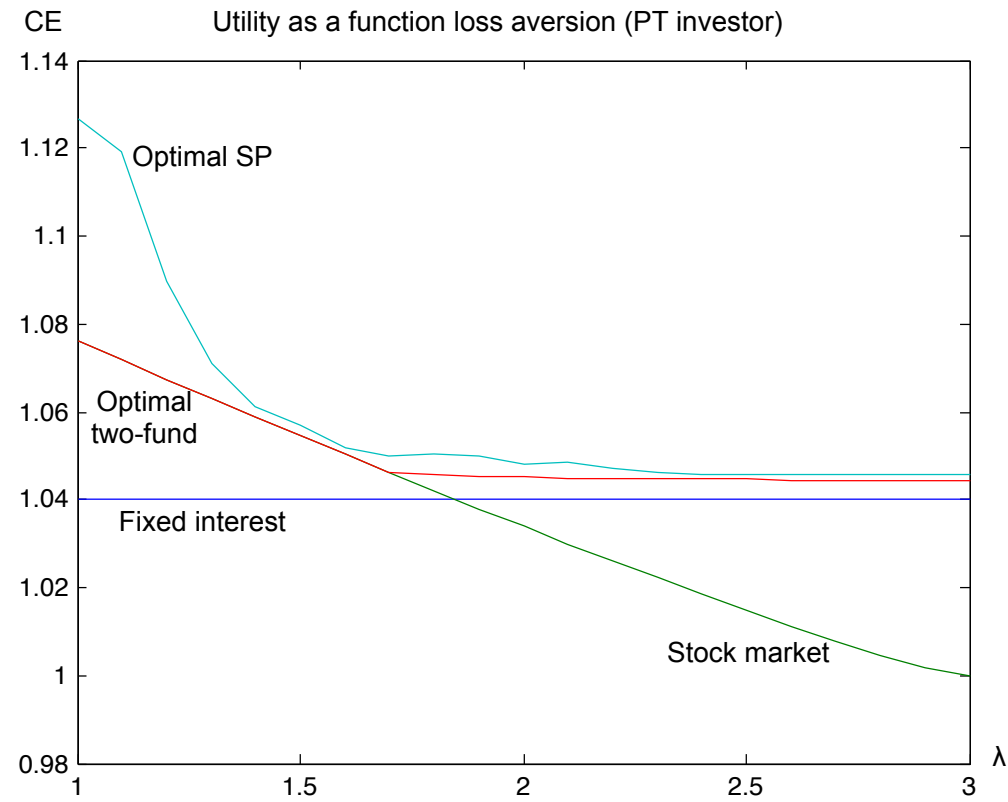

Figure 6: Prospect utility (as measured by the certainty equivalent interest rate) of stock, risk-free assets (4\% return), optimal two-fund portfolios and optimally structured products. We have varied the loss aversion $\lambda$ of the investor, while $\alpha=\beta=0.88$ were fixed. 


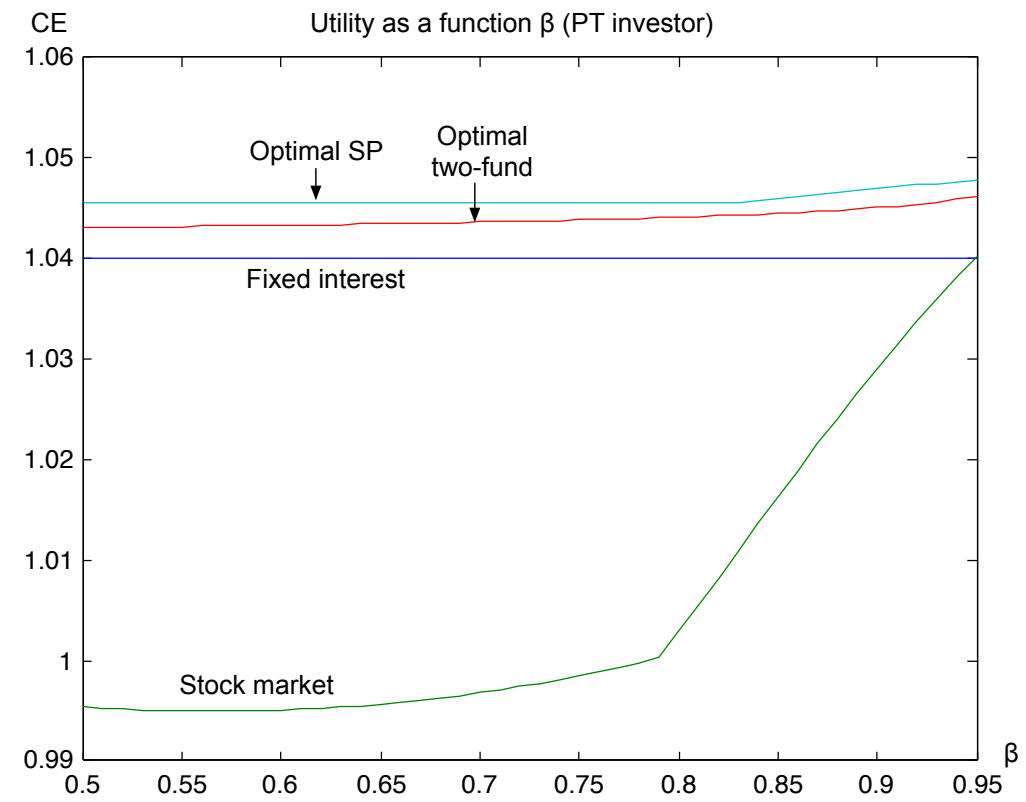

Figure 7: Prospect utility (as measured by the certainty equivalent interest rate) of stock, risk-free assets (4\% return), optimal two-fund portfolios and optimally structured products. We have varied the degree of risk seeking in losses beta of the investor, while $\alpha=0.88$ and $\lambda=2.25$ were fixed. 
be explained by the impact of the risk seeking behavior in losses. A high degree of loss aversion mitigates this effect (as the gambles corresponding to the return distribution of the structured products are not pure loss gambles), while in the loss-neutral case, this risk-seeking behavior substantially increases the utility gain by a jump in the payoff function. The overall utility gain is, in our example, equivalent to around $1 \%$ gain in returns for a normal loss aversion of approximately 2. This gain is comparable and even sometimes larger than typical implicit fees for simple types of structured products.

Thus, loss aversion and (even more so) gambling to avoid sure losses are motives that cannot be served well by a standard portfolio composed of the risk-free rate and the underlying. Here structured products can be an attractive alternative.

\subsection{Incorrect beliefs}

We might also consider factors other than simply risk preferences. One such factor is that agents do not apply market beliefs in their optimization. This may be the case because agents have different opinions than the market, i.e. they believe they are better than the market in anticipating the future asset returns. In a simple model one can compare the agents beliefs about the mean return and the variance of returns of the market. If an agent beliefs the market is more likely going up than is reflected in the market prices, we say he is optimistic (and in the opposite case pessimistic). If an agent's estimation of market volatility is smaller than that of the market we say he is overconfident in his expectations. A different case in which agents do not apply market beliefs in their optimization arises when they agree to the probabilities of market returns but react in a biased way to those probabilities. In particular, Kahneman \& Tversky (1979) found that 
individual decision makers overweigh small probabilities in their decisions ${ }^{14}$. Unlikely but still possible events get larger decision weight than their probability reflects. As an effect agents which have a strong degree of probabilty weighting believe that the market volatility is higher than reflected in the market prices (see Example 2).

In order to analyze incorrect beliefs we have to change our model. Fortunately, doing so turns out to be easy: let us denote the probability applied by the investor ${ }^{15}$ by $\tilde{p}$; then, his optimization problem becomes

$$
\text { Maximize } U(y):=\int_{\mathbb{R}} u(y(x)) \tilde{p}(x) d x
$$

in $L_{\ell p}^{1}(\mathbb{R})$ subject to

$$
\int_{\mathbb{R}} y(x) \ell(x) p(x) d x=R
$$

Defining $w(x):=p(x) / \tilde{p}(x)$ and $\tilde{\ell}(x):=w(x) \ell(x)$, we can transform this problem to

$$
\text { Maximize } U(y):=\int_{\mathbb{R}} u(y(x)) \tilde{p}(x) d x
$$

subject to

$$
\int_{\mathbb{R}} y(x) \tilde{\ell}(x) \tilde{p}(x) d x=R
$$

This new problem can now be solved in the same way as before. The following two examples illustrate the optimally structured product and its utility gain over the classical portfolio for the case of incorrect beliefs.

Example 1 (Optimism and pessimism). Let $p$ and $\tilde{p}$ be normal distributions. Then an optimistic investor chooses an outperformance product, i.e.

\footnotetext{
${ }^{14}$ Probability weighting can explain why people participate in the national lottery even though they know that the probability of winning is too small tojustify the cost of the lottery ticket.

${ }^{15}$ Tversky \& Kahneman (1992) introduce a probability weighting function $w:[0,1] \mapsto$ $[0,1]$. In this case the incorrect beliefs applied in the utility maximization are $\tilde{p}=w(p)$.
} 
a structured product that increases more than the market while a pessimistic investor chooses a bear tracker, i.e. a produc that gains when the market loses.

Example 2 (Over- and underconfidence). Let $p$ and $\tilde{p}$ be normal distributions. We say an investor is over- (under-) confident if he or she has a too narrow (wide) probability estimate, i.e., $\operatorname{var} p>\operatorname{var} \tilde{p}$, but $\mathbb{E}(p)=\mathbb{E}(\tilde{p})$. The (subjective) utility gain (again measured in fixed-interest certainty equivalent units) can easily reach several percentage points, as illustrated in Table 3 , in which the mean is now estimated correctly, but the variance is misestimated. If the volatility is $20 \%$, but it is estimated by the investor as $25 \%$, then structured products allow for an improvement of the subjective utility by $2.05 \% .{ }^{16}$ Similarly, if the volatility is estimated as $15 \%$, the subjective utility gain is $2.44 \%$, so both under- and overconfidence can make structured products look attractive.

Table 3: Improvement by a structured product for CRRA investors with incorrect beliefs, compared to classical portfolios.

\begin{tabular}{lc} 
Investor type & $\begin{array}{c}\text { Improvement over } \\
\text { classical portfolio }\end{array}$ \\
\hline Pessimist (expected return 9\% below market return) & $1.1 \%$ \\
Underconfident (expected volatility 5\% above market volatility) & $2.05 \%$ \\
Overconfident (expected volatility 5\% below market volatility) & $2.44 \%$
\end{tabular}

In summary, the explanations of the high demand for structured investment products based on behavioral preferences and biases, such as incorrect beliefs, are the most convincing.

\footnotetext{
${ }^{16}$ This case resembles the situation of probability overweighting, as mentioned above.
} 


\section{Conclusion and outlook}

In this paper, we have discussed whether it is possible to explain the demand for structured investment products in a utility maximization framework. The answer we have found is two-fold. There is no evidence that the classical von Neumann-Morgenstern expected utility with correct beliefs can explain this demand. However, for prospect theory utilities with loss aversion and risk taking to avoid sure losses or for non-rational beliefs, some structured products can be considered utility-optimizing. To be precise, the utility improvements delivered by optimally structured products:

- are almost zero for expected utility with correct beliefs;

- can be substantial and higher than typical fees for prospect theory investors with correct beliefs; and

- can be substantial and higher than typical fees for investors with incorrect beliefs.

This is a rather pessimistic view of structured products, which after all are an important industry in many countries. Before giving policy advice based on our results, we suggest also looking into other possible reasons for the existence of structured products.

One possible route for further research is analyzing structured products in a portfolio context. Perhaps some structured products make sense in a portfolio model with several mental accounts. If the other needs of the investor are met in a different mental account, a structured product could serve specific needs that are not captured by an all-encompassing utility maximization in a single mental account. Some attractive examples in this respect can be found in Shefrin \& Statman (2000). The other route is to look into decision models, other than prospect theory, that depart more substantially from expected utility. Examples are the rank dependent utility 
model of Quiggin (1993) and the SP/A decision model introduced by Lopes (1987) and Lopes \& Oden (1999). Indeed, the paper of Polkovnichenko (2005) demonstrates interesting differences in the asset allocation of rank dependent utilities, expected utility and cumulative prospect theory. The implications of these differences for structured products are worth exploiting. Moreover, the examples of Shefrin (2008) and in Shefrin \& Statman (2000) for structured products in a SP/A framework is very promising for a general utility maximization analysis of structured products based on SP/A theory.

\section{Acknowledgement}

We thank Nicole Branger, Bernhard Eckwert, Michel Habib, Wolfgang Härdle, Frank Riedel, Hersh Shefrin and an anonymous referee of this journal for their interesting discussions about this work. We are grateful to Derivative Partners and to Meike Bradbury and Blerim Tahiraj for providing statistical data on structured products. We particularly thank the anonymous referee for helpful suggestions for improving the focus of this paper. Support from the National Centre of Competence in Research "Financial Valuation and Risk Management" (NCCR FINRISK), Project A1, from "Behavioural and Evolutionary Finance", and from the University Priority Program "Finance and Financial Markets" of the University of Zürich is gratefully acknowledged.

\section{References}

N. Barberis \& R. Thaler (2003). 'A survey of behavioral finance'. In G. Constantinides, M. Harris, \& R. M. Stulz (eds.), Handbook of the Economics of Finance, vol. 1, chap. 18, pp. 1053-1128.

N. Benet, et al. (2006). 'Gains from structured product markets: The case of reverse-exchangeable securities (RES)'. Journal of Banking and Finance 
30(1):111-132.

C. Bernard, et al. (2007). 'Optimal Design of Structured Products and the Role of Capital Protection'. working paper.

P. L. Bossaert (2002). The paradox of asset pricing. Princeton University Press.

N. Branger \& B. Breuer (2007). 'The optimal demand for retail derivatives'. Working paper, University of Münster .

W. Breuer \& A. Perst (2007). 'Retail banking and behavioral financial engineering: the case of structured products'. Journal of Banking and Finance 31:827-844.

P. Carr \& D. Madan (2001). 'Optimal positioning in derivative securities'. Quantitative Finance 1(1):19-37.

C. Chiarella, et al. (2007). 'Heterogeneous expectations and speculative behavior in a dynamic multi-asset framework'. Journal of Economic Behavior \& Organization 62(3):408-427.

D. Duffie (2001). Dynamic Asset Pricing Theory. Princeton University Press.

A. Gerber \& T. Hens (2006). 'Modelling Alpha-Oppurtunities Within the CAPM'. Tech. rep., SSRN working paper.

C. Gollier (2004). The economics of risk and time. MIT Press.

A. Grünbichler \& H. Wohlwend (2005). 'The Valuation of Structured Products: Empirical Findings for the Swiss Market'. Financial Markets and Portfolio Management 19(4):361-380.

B. Henderson \& N. Pearson (2011). 'The dark side of financial innovation'. Journal of Financial Economics 100:227-247. 
B. J. Henderson \& N. D. Pearson (2007). 'Patterns in the Payoffs of Structured Equity Derivatives'. SSRN working paper.

R. A. Jarrow \& D. B. Madan (1997). 'Is Mean-Variance Analysis Vacuous: Or was Beta Still Born?'. European Finance Review 1:15-30.

D. Kahneman \& A. Tversky (1979). 'Prospect Theory: An Analysis of Decision Under Risk'. Econometrica 47:263-291.

D. Kramkov \& W. Schachermayer (1999). 'The asymptotic elasticity of utility functions and optimal investments in incomplete markets'. The Annals of Applied Probability 9(3):904-950.

H. E. Leland (1980). 'Who Should Buy Portfolio Insurance?'. The Journal of Finance XXXV(2):581-594.

L. L. Lopes (1987). 'Between hope and fear: The psychology of risk'. Advances in Experimental Social Psychology 20(255-295).

L. L. Lopes \& G. C. Oden (1999). 'The Role of Aspiration Level in Risky Choice: A Comparison of Cumulative Prospect Theory and SP/A Theory'. Journal of Mathematical Psychology 43(2):286-313.

R. Merton (1971). Continuous Time Finance. Basil Blackwell.

S. R. Pliska (1984). 'A stochastic calculus model of continuous trading: optimal portfolios'. Discussion Paper.

V. Polkovnichenko (2005). 'Household Porfolio Diversification: A case for rank dependent preferences'. The Review of Financial Studies 18(4):14671502.

J. Quiggin (1993). Generalized Expected Utility Theory: The Rank Dependent Model. Kluwer: Norwell MA, USA. 
M. O. Rieger (2009). Optionen, Derivate und strukturierte Produkte (Options, Derivatives and Structured Products). NZZ-Verlag and SchäfferPoeschel.

M. O. Rieger (2011). 'Co-monotonicity of optimal investments and the design of structural financial products'. Finance and Stochastics 15(1):2755.

M. O. Rieger (2012). 'Optimal financial investments for non-concave utility functions'. Economics Letters 114:239-240.

A. D. Roy (1952). 'Safety First and the Holding of Assets'. Econometrica 431:431-450.

M. Rubinstein (1974). 'An Aggregation Theorem for Securities Markets'. Journal of Financial Economics 1(3):225-44.

H. Shefrin (2008). A behavioral approach to asset pricing (2nd edition). Elsevier.

H. Shefrin \& M. Statman (1993). 'Behavioral Aspects of the Design and Marketing of Financial Products'. Financial Management 22(2):123-134.

H. Shefrin \& M. Statman (2000). 'Behavioral Portfolio Theory'. Journal of Financial and Quantitative Analysis 35(2).

Stoimenov \& Wilkens (2005). "Are structured products "fairly" priced? an analysis of the German market for equity-linked instruments'. Journal of Banking and Finance 29(12):2971-2993.

M. Szymanowska, et al. (2007). 'Reverse Convertible Bonds Analyzed'. SSRN working paper .

A. Tversky \& D. Kahneman (1992). 'Advances in Prospect Theory: Cumulative Representation of Uncertainty'. Journal of Risk and Uncertainty 5:297-323. 
J. von Neumann \& O. Morgenstern (1944). Theory of Games and Economic Behavior. Princeton University Press, Princeton, NJ.

M. Wallmeier \& M. Diethelm (2008). 'Market Pricing of Exotic Structured Products: The Case of Multi-Asset Barrier Reverse Convertibles in Switzerland'. Tech. rep., University of Fribourg.

S. Wilkens, et al. (2003). 'The Pricing of Structured Products - An Empirical Investigation of the German Market'. The Journal of Derivatives 11(1):55-69.

S. Wilkens \& P. Stoimenov (2007). 'The pricing of leverage products: An empirical investigation of the German market for 'long' and 'short' stock index certificates'. Journal of Banking and Finance 31:735-750.

\section{A Mathematical proofs}

\section{A.1 Computation of the optimal Structure Product for CRRA utility.}

Let $u(x):=\frac{1}{\alpha} x^{\alpha}$ with $\alpha<1, \alpha \neq 0$, then $v(x):=u^{\prime}(x)=x^{\alpha-1}$ and $v^{-1}(z)=z^{\frac{1}{\alpha-1}}$. Therefore, recalling (3), the optimal structured product is given by

$$
y(x)=(\lambda \ell(x))^{-\frac{1}{1-\alpha}} .
$$

We can compute $\lambda$ explicitly, if we use the constraint (2):

$$
\int(\lambda \ell(x))^{-\frac{1}{1-\alpha}} \ell(x) p(x) d x=R,
$$

which can be resolved to

$$
\lambda=\left(\frac{R}{\int \ell(x)^{\frac{\alpha}{\alpha-1}} p(x) d x}\right)^{\alpha-1} .
$$


A.2 Computation of the optimally structured product in the CARA case 31

All together, we obtain

$$
y(x)=\frac{C}{\ell(x)^{\frac{1}{1-\alpha}}}, \text { where } C:=\frac{R}{\int \ell(x)^{\frac{\alpha}{\alpha-1}} p(x) d x} .
$$

In the case of the CAPM, we obtain

$$
y(x)=\frac{C}{(a-b x)^{\frac{1}{1-\alpha}}} .
$$

\section{A.2 Computation of the optimally structured product in the CARA case}

Let $u(x):=-\frac{1}{\alpha} e^{-\alpha x}$. Then, $v(x):=u^{\prime}(x)=e^{-\alpha x}$ and $v^{-1}(z)=-\frac{1}{\alpha} \ln z$. Thus,

$$
y(x)=-\frac{1}{\alpha} \ln (\lambda \ell(x)) .
$$

Again, we can compute $\lambda$ explicitly, if we use 2, i.e.,

$$
\int y(x) \ell(x) p(x) d x=R
$$

The left hand side can be computed as follows:

$$
\begin{aligned}
\int y(x) \ell(x) p(x) d x & =-\frac{1}{\alpha} \int \ln (\lambda \ell(x)) p(x) \ell(x) d x \\
& =-\frac{1}{\alpha} \int(\ln (\lambda)+\ln (\ell(x))) p(x) \ell(x) d x \\
& =-\frac{1}{\alpha} \ln (\lambda)-\frac{1}{\alpha} \int \ln (\ell(x)) p(x) \ell(x) d x
\end{aligned}
$$

Thus, we obtain

$$
\lambda=e^{-\alpha R-\int p(x) \ell(x) \ln (\ell(x) d x} .
$$

The optimally structured product is therefore given by

$$
y(x)=R-\frac{1}{\alpha}\left(\ln \ell(x)-\int p(x) \ell(x) \ln (\ell(x)) d x\right) .
$$

And in the case of the CAPM, we obtain

$$
y(x)=R-\frac{1}{\alpha}(\ln (a-b x)-C),
$$

where $C:=\int p(x)(a-b x) \ln (a-b x) d x$. 